\title{
Posterior reversible encephalopathy syndrome: pitfalls of T2 shine through in MRI
}

\author{
Taichi Omachi ${ }^{1,2}$,Tomohiko Shimo ${ }^{1}$, Takahide Nakano ${ }^{1}$, Masaya Takahashi ${ }^{1}$ and Junji Takaya ${ }^{1 *}$ \\ ${ }^{1}$ Department of Pediatrics, Kawachi General Hospital, Osaka, Japan \\ ${ }^{2}$ Department of Pediatrics, Kansai Medical University, Osaka, Japan
}

\begin{abstract}
A 6-year-old Japanese girl was presented to us with recurrent abdominal pain and vomiting for 1 week without altered mental status and was admitted to the hospital diagnosed with constipation and dehydration. On the evening of her admission, the child had markedly elevated blood pressure (151/98 mmHg) that was followed by seizure and sudden dysarthria. She had aphasia, dysarthria and right hemi-convulsion for 5 min. Imaging studies showed hyper-intense signaling on T2W images, as well as hyper-iso-intense signaling on fluid attenuated inversion recovery images with restriction of diffusion seen involving the cortex of the occipito-parietal region bilaterally, consistent with posterior reversible encephalopathy syndrome (PRES). First, the patient was treated with steroid pulse. Investigations yielded normal values for urinalysis and central spinal fluid. Systolic blood pressure persisted at 140 to $150 \mathrm{mmHg}$. Methylprednisolone was administered for 3 days intravenously. The patient was treated with nicardipine hydrochloride and showed improvement. Right occipito-parietal areas of high diffusion-weighted imaging (DWI) signal intensity was seen with apparent diffusion coefficients (ADCs) values that were iso- and partial hyper-intense signaling. Vasogenic edema shows T2 shine through in ADC map. ADC map in addition to performing DWI is important to the diagnosis of PRES.
\end{abstract}

\section{Introduction}

Posterior reversible encephalopathy syndrome (PRES) usually presents with rapid onset of symptoms, including headache, seizure, altered level of consciousness, and visual disturbance [1]. The disorder is common in patients with renal disease and often coexists with conditions such as hypertension, exposure to immunosuppressive drugs. The pathophysiological mechanisms underlying PRES are not fully understood; however, the most common mechanism is related to increasing blood pressure causing failure of brain autoregulation.

\section{Case Report}

A 6-year-old Japanese girl was presented to us with recurrent abdominal pain and vomiting for 1 week without altered mental status, cough, or any other symptoms. She was admitted to the hospital and diagnosed with constipation and dehydration; she received supportive intravenous fluid therapy. Her developmental history was compatible with her age. Her weight was $22 \mathrm{~kg}$; height, $121 \mathrm{~cm}$; pulse rate, 150 beats $/ \mathrm{min}$; temperature, $37.2^{\circ} \mathrm{C}$; and blood pressure, $110 / 70 \mathrm{~mm} \mathrm{Hg}$. An abdominal examination revealed normal bowel sounds and her initial neurologic examination was unremarkable. Laboratory tests showed the following results: hemoglobin concentration, $16.2 \mathrm{~g} / \mathrm{dL}$; white blood cell count, $15.6 \times 10^{9} / \mathrm{L}$ with $84 \%$ neutrophils; platelet count, 46.3 $\times 10^{10} / \mathrm{L}$; creatinine level, $0.26 \mathrm{mg} / \mathrm{dL}$; sodium, $133 \mathrm{mEq} / \mathrm{L}$; potassium, $4.6 \mathrm{mEq} / \mathrm{L}$; serum keto-body, $2.3 \mathrm{mmol} / \mathrm{L}$; and C-reactive protein level, $0.83 \mathrm{mg} / \mathrm{dL}$. Coagulation studies revealed normal activated partial thromboplastin time and fibrinogen, while the prothrombin time was slightly elevated at 1.23 (normal range $0.91-1.14$ ) and the fibrin degradation product was elevated at $13.1 \mu \mathrm{g} / \mathrm{mL}$ (normal range $<5.0 \mu \mathrm{g}$ / $\mathrm{mL}$ ). After discharge, serum M pneumonia (PH) antibody was found to be positive (titer 1:160), suggestive of acute infection.

On the evening of her admission, the child had markedly elevated blood pressure $(151 / 98 \mathrm{mmHg})$ that was followed by seizure and sudden dysarthria (loss of vision). She had aphasia, dysarthria and right hemiconvulsion for $5 \mathrm{~min}$, which recovered after a while. On examination, she was drowsy, had a Glasgow Coma Scale score of 10, and was responding to verbal stimuli. Brain computed tomography showed no remarkable findings. The next morning, the patient complained of visual disturbance. Imaging studies showed hyper-intense signaling on $\mathrm{T} 2 \mathrm{~W}$ images (Figure 1A), as well as hyper-isointense signaling on fluid attenuated inversion recovery images (Figure 1B) with restriction of diffusion seen involving the cortex of the occipito-parietal region bilaterally, suggesting PRES. Right occipito-parietal areas of diffusionweighted imaging (DWI) signal hyperintensity (Figure 1C) were seen with apparent diffusion coefficients (ADCs) values that were partial hyper and isodense signaling (Figure 1D). On the other hand, left occipito-parietal high-intense signaling on T2W images were seen with hyper-isointense ADC scores suggesting vasogenic edeme. Magnetic resonance angiography showed no remarkable findings.

Based on these findings, the diagnosis of PRES was made. First, the patient was treated with steroid pulse. Investigations yielded normal values for urinalysis and central spinal fluid. Systolic blood pressure persisted at 140 to $150 \mathrm{mmHg}$. Methylprednisolone was administered for 3 days intravenously. The patient was treated with nicardipine hydrochloride and showed improvement.

The most characteristic imaging pattern in PRES is the presence of edema involving the white matter of the posterior portion of both

${ }^{*}$ Correspondence to: Junji Takaya, Department of Pediatrics, Kawachi General Hospital, 1-31 Yokomakura, Higashiosaka, Osaka, 578 0954, Japan, Tel: +8172-965- 0731; E-mail: takaya@kawati.or.jp

Key words: ADC map, PRES

Received: June 21, 2019; Accepted: June 26, 2019; Published: July 01, 2019 


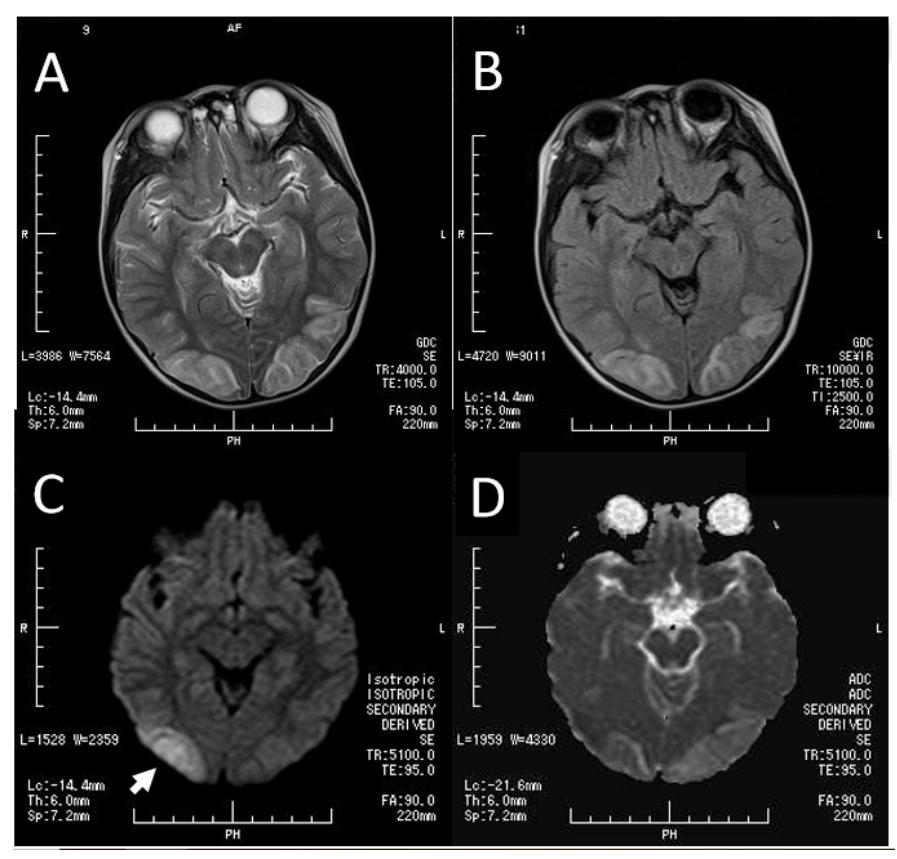

Figure 1. A. Magnetic resonance imaging of the brain shows a T2-weighted high-intensity signal in the bilateral parieto-occipital region; B. Fluid-attenuated inversion recovery magnetic resonance imaging shows mild edema in the bilateral parieto-occipital region; C. Diffusion-weighted imaging confirms moderately restricted diffusion focally in the right parieto-occipital edema (arrow); D. Apparent diffusion coefficient map confirms nonrestricted diffusion. ADC values were normal or high intensity (T2 shine through) in right parieto-occipital regions

cerebral hemispheres, especially in the parieto-occipital regions. Both PRES and cerebral infarcts present with abnormal hyperintensities on fluid-sensitive sequences, the distribution of signal abnormalities is usually different in both disorders. DWI is typically positive in cerebral infarcts, whereas DWI may or may not be positive in PRES [2]. It is not possible to differentiate between cytotoxic and vasogenic edema on T2-weighted images. In the present case, the areas of high DWI signal intensity in right parieto-occipital regions were seen with $\mathrm{ADC}$ values that were normal or partial high, which were consistent with T2 shine-through effect [4]. T2 shine through refers to high signal on DWI images that is not due to restricted diffusion. ADC values allow the differentiation of vasogenic edema from cytotoxic edema [3]. On the other hand, areas of normal DWI signal intensity in left parieto-occipital regions were seen with ADC values that were normal or high, which were consistent with vasogenic edema. Creating an ADC map in addition to performing DWI is important to rule out the diagnosis of PRES. Extrapulmonary complications of Mycoplasma (M.) pneumoniae infection involving the central nervous system, such as infarction, encephalitis, and Guillain-Barré syndrome, are not rare [4]. M. pneumoniae infection and resultant inflammation may have contributed to the generation of PRES [5].

\section{Acknowledgements}

We thank Dr. Akira Murasawa, Kazutami Nakao, Masahiro Mukai for their fruitful discussion. We also thank the radiologist technicians at Kawachi General Hospital for their technical assistance.

\section{Conflict of Interest}

The authors declare that there is no conflict of interest regarding the publication of this article.

\section{References}

1. Fugate JE, Rabinstein AA (2015) Posterior reversible encephalopathy syndrome: clinical and radiological manifestations, pathophysiology, and outstanding questions. Lancet Neurol 14: 914-925. [Crossref]

2. Mukherjee P, McKinstry RC (2001) Reversible posterior leukoencephalopathy syndrome: evaluation with diffusion-tensor MR imaging. Radiology 219: 756-765. [Crossref]

3. Covarrubias DJ, Luetmer PH, Campeau NG (2002) Posterior reversible encephalopathy syndrome: prognostic utility of quantitative diffusion-weighted MR images. AJNR Am J Neuroradiol 23: 1038-1048. [Crossref]

4. Kastrup O, Schlamann M, Moenninghoff C, Forsting M, Goerickes S (2015) Posterior Reversible Encephalopathy Syndrome: The Spectrum of MR Imaging Patterns. Clin Neuroradiol 25: 161-171. [Crossref]

5. Ramgopal A, Thavamani A, Ghori A (2018) Association between posterior reversible encephalopathy syndrome and Mycoplasma pneumonia infection. J Pediatr Neurosci 13: 109-111. [Crossref]

Copyright: (C2019 Omachi T. This is an open-access article distributed under the terms of the Creative Commons Attribution License, which permits unrestricted use, distribution, and reproduction in any medium, provided the original author and source are credited. 